\title{
High energy physics grows tenfold
}

\author{
from Robert Walgate
}

THE first ever controlled collisions of fundamental particles at centre of mass energies of over $540 \mathrm{GeV}$ - ten times higher than previously achieved, and an energy at which spectacular physics is expected - are beginning to be observed at the new proton antiproton collider at the European centre for nuclear research (CERN), even before the machine is out of the hands of its constructors.

"We take data while the engineers take their coffee breaks", Carlo Rubbia, leader of one of the two experiments now in place, said last week. Rubbia - a well-loved Italian-American maverick of high-energy physics - in fact proposed the whole idea of the collider, in which bunches of protons at $270 \mathrm{GeV}$ collide with oppositely moving bunches of antiprotons. The proposal came in, in a report at the 1976 Aachen neutrino conference titled "Looking for the intermediate vector boson with existing accelerators". He has not been slow to reap the rewards now the machine is in place. So far his experiment (UA1) has recorded some 20,000 events, but no intermediate vector boson.

On the other side of the collider (which is in fact the super proton synchrotron modified to take colliding beams) is experiment UA5, headed by Dr John Rushbrooke of the University of Cambridge with professors Klaus Bockmann of Bonn, Jacques Lemonne of Brussels and Gösta Eksborg of Stockholm and their groups. UA5 has taken 1,500 pictures (one of which appears below) with another 10,000 expected in the next month.

The events of UA 1 and UA 5 together are only the first scratching of the surface of physics at these exalted energies, but

Robert Walgate is Chief European Correspondent of Nature. already there are some indications of what may or may not be expected.

The physics needs to be seen in relation to two quite separate classes of prediction. On the one hand, there are some peculiar data from cosmic ray experiments - a handful of "Centauro" events and others - which indicate that there may be a substantial change in physics at energies close to, or perhaps somewhat above, the energies of the new collider ${ }^{1}$. On the other, there are the predictions of the SalamWeinberg unified gauge theory of the weak and electromagnetic interactions, which has been well-tested at lower energies in electron and neutrino scattering experiments.

This theory is the backbone of most current attempts at a "grand unification" of all the forces, and it predicts the existence of three intermediate vector bosons $\left(\mathrm{Z}^{\circ}, \mathrm{W}^{+}\right.$, and $\left.\mathrm{W}^{-}\right)$at masses of around $80 \mathrm{GeV}$. These should be created at collider energies, and should be seen to decay as predicted by the theory. The theory also predicts new scalar particles called Higgs' particles, but the masses of these are, generally speaking, undetermined and the particles may or may not turn up in collider experiments.

So far there are no data from CERN on the intermediate vector bosons, but that is not unexpected. Estimates suggest that the IVBs will only be produced in small numbers, and too few events have been collected so far to expect one among them. Finding the bosons also depends on increasing the luminosity of the collider (effectively the number of collision per second per unit interaction cross section) by orders of magnitude beyond those presently being reached at CERN. Design luminosity was $10^{30} \mathrm{~s}^{-1} \mathrm{~cm}^{2}$; the present achievement is $2 \times 10^{25}$. A luminosity of $10^{29}$ would give Rubbia about $10 \mathrm{Ws}$ and
1-2 $Z^{\circ}$ a day, he estimates, allowing for his detector efficiency.

CERN machine physicists have however not yet attempted to "tune" the collider to give its maximum luminosity. Two effectively independent factors of 50 could be obtained relatively easily, it is believed, which would bring the machine within a factor of 2 of a luminosity of $10^{29}$. The remaining factor of 2 might be made up within a year, machine physicists feel, with $10^{30}$ remaining so far a very distant goal.

More easy to check are the cosmic ray results. These indicate that some extraordinary and inexplicable interactions may set in at centre of mass energies of the order of $1,000 \mathrm{GeV}$ (This is around twice the collider energy, but the cosmic ray experiments have a detector threshold at about that level, Rushbrooke believes, so the physical threshold may be lower.) These Centauro events - of which only five or so have been observed, cosmic ray intensities being so low at this energy - show the production of very large numbers of charged particles (track multiplicity) and a mysterious near absence of neutral pions. Also the average transverse momentum of these and other high energy cosmic ray events rises with multiplicity, something quite unknown in lower energy data.

However, so far neither UAI or UA5 have found any clear indication of these phenomena at the collider - though exact results await more precise data analysis.Confirmation of the cosmic ray results may have to wait for higher statistics - or for a collider of even higher energy, such as the $2,000 \mathrm{GeV}$ centre of the mass energy collider being planned at Fermilab near Chicago for action in 1984 or 1985 (Ronald Reagan willing).

1. Latters, C.M.G., Fujimoto, Y., \& Hasegawa, S. Physics Reports 65, 3 (1980).

One of the first $540 \mathrm{GeV}$ p-p events to be recorded in the Cambridge-Bonn-Brussels-Stockholm streamer chamber (experiment UA5), showing clear forward and backward cones of tracks. The streamer chamber is the only detector on the collider to record particle tracks clearly close to the interaction point. It will provide a first survey of the physics of interactions at these new energies.

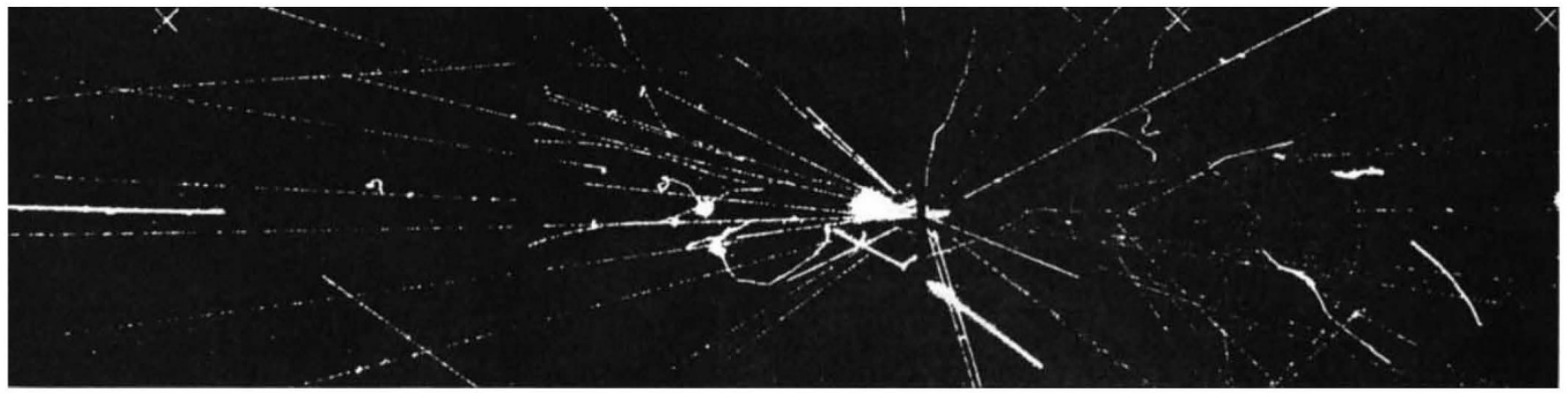

